\title{
MASSENZIO (Marcello), Sacré et identité ethnique. Frontières et ordre du monde
}

Paris, Cahiers de l'Homme, Éditions de l'E.H.E.S.S., 1999, 183 p. (préface de Marc Augé. Traduit de l'italien par Federica Giardini et Valérie Giardini. Traduction revisée par Giordana Charuty) (bibliogr., index)

\section{Carmen Bernand}

\section{(2) OpenEdition \\ 12 Journals}

Édition électronique

URL : http://journals.openedition.org/assr/20329

DOI : 10.4000/assr.20329

ISSN : $1777-5825$

\section{Éditeur}

Éditions de l'EHESS

\section{Édition imprimée}

Date de publication : 31 décembre 2000

Pagination : 106-108

ISBN : 2-222-96698-1

ISSN : 0335-5985

\section{Référence électronique}

Carmen Bernand, «MASSENZIO (Marcello), Sacré et identité ethnique. Frontières et ordre du monde », Archives de sciences sociales des religions [En ligne], 112 | octobre-décembre 2000, document 112.34, mis en ligne le 19 août 2009, consulté le 21 septembre 2020. URL : http://journals.openedition.org/ assr/20329; DOI : https://doi.org/10.4000/assr.20329

Ce document a été généré automatiquement le 21 septembre 2020.

(c) Archives de sciences sociales des religions 


\section{MASSENZIO (Marcello), Sacré et identité ethnique. Frontières et ordre du monde}

Paris, Cahiers de l'Homme, Éditions de l'E.H.E.S.S., 1999, 183 p. (préface de Marc Augé. Traduit de l'italien par Federica Giardini et Valérie Giardini. Traduction revisée par Giordana Charuty) (bibliogr., index)

\section{Carmen Bernand}

\section{RÉFÉRENCE}

MASSENZIO (Marcello), Sacré et identité ethnique. Frontières et ordre du monde, Paris, Cahiers de l'Homme, Éditions de l'E.H.E.S.S., 1999, 183 p. (préface de Marc Augé. Traduit de l'italien par Federica Giardini et Valérie Giardini. Traduction revisée par Giordana Charuty) (bibliogr., index)

1 Un des intérêts de ce livre est l'interprétation de matériaux ethnographiques dans la perspective de l'histoire des religions, telle qu'elle s'est développée en Italie. M.M. présente l'œuvre de Raffaelle Pettazzoni, Angelo Brelich et Ernesto de Martino pour en dégager les principales notions qu'il utilisera dans un second temps pour analyser des rituels pratiqués en Nouvelle-Guinée. Un premier chapitre théorique est consacré à la portée de l'histoire des religions, telle qu'elle a été définie par ces trois auteurs. Tous ont en commun le refus de réduire le religieux à d'autres phénomènes, économiques ou politiques, par exemple. Ils revendiquent l'autonomie de la religion et du mythe sans pour autant postuler l'existence d'un sacré a priori, à la manière de Mircea Éliade. Le sacré est la création de l'homme, mais possède ses propres règles, ses propres fonctions et sa propre signification. L'approche dialectique entre le sacré et le profane est présentée ici dans une perspective qui n'est pas aussi éloignée que l'on pourrait croire de Durkheim. Mais contrairement au sociologue français, qui fait de l'émotion la source première du sentiment religieux, Pettazzoni insiste sur l'importance de l'expérience 
humaine dans la construction du sacré, qui est conscience et acceptation de la condition humaine.

2 Les réflexions d'Angelo Brelich sur le sacré adoptent une perspective différente. "Qu'est-ce donc qui, dans certaines civilisations, confère une importance religieuse à ce qui dans d'autres civilisations peut ne pas en avoir?». Les facteurs déterminant le caractère sacré de certains éléments dépendent en général, du besoin qu'a l'homme de maitriser la réalité phénoménale. Le sacré doit se soumettre à une règle fondamentale, celle d'imposer des limites précises à ses manifestations, clôturer son espace. La « concentration du sacré » qui est à l'œuvre dans l'offrande des prémices, permet de transformer le produit des récoltes en utilisation profane. L'autre point essentiel est l'attribution d'un sens à la réalité, qui est pour l'homme le règne de la pure contingence. Pour fonder un ordre humain soumis à des normes il faut arracher d'abord la réalité à sa contingence : c'est le propre des mythes qui partent toujours d'une fondation. Tout prend un sens dont la clé se trouve dans le temps des origines et tout devient nécessaire.

Comme Pettazzoni et Brelich, Ernesto de Martino pense que les phénomènes religieux sont une production particulière de l'homme, que le positivisme a appauvrie. Le sacré représente l'altérité absolue par rapport à l'humain. Pour de Martino, la tâche essentielle de l'histoire des religions doit porter sur la genèse du sacré chez l'individu. La dimension du sacré comprend la perte de la présence et sa restauration. En tant qu'altérité absolue, le sacré implique donc la négation de l'histoire, ou la déshistorisation. Comment peut-on nier le devenir? Par répétition constante d'un contenu qui nie tout changement. La déshistorisation religieuse, dans la sphère du sacré, est produite par des institutions culturelles. Pour reconstituer le processus de la dynamique culturelle de la crise et de la restauration de la présence, il faut une démarche pluridisciplinaire. Mais l'importance de la crise dans son œuvre - qui est aussi celle d'un ethnologue aux prises avec le terrain - le porte à relier l'histoire des religions à la psychopathologie et à la psychologie. La religion n'est pas une croyance arriérée mais «la seule voie possible pour protéger les groupes humains d'une crise existentielle et pour restaurer une certaine conscience de l'humanité ainsi qu'une possibilité d'agir selon des valeurs instaurées par l'homme ».

4 Armé des concepts élaborés par l'histoire des religions, notamment, ceux de crise existentielle, de déshistorisation, de transfiguration et d'identification, M.M. se penche sur un ensemble rituel de Nouvelle-Guinée qui met en œuvre, entre autres choses, un massacre de cochons domestiques. L'examen de ces matériaux ethnographiques s'inspire d'une première question fondamentale, celle qui concerne la frontière entre les humains et les animaux. Après avoir montré la différence des attitudes à l'égard de la mise à mort des animaux chez les chasseurs et chez les éleveurs, il choisit d'explorer la façon dont un peuple, les Fouyoughé, qui domestique les cochons et leur accorde un statut quasi-humain, parvient à renverser les interdits qui portent sur leur transformation en nourriture, et à les faire massacrer, lors d'un rituel appelé le gâbé. Nous avons là une situation de "crise », déclenchée par la transgression qui implique la tuerie des animaux domestiques, considérés comme un bien propre, tuerie pourtant nécessaire pour activer le réseau d'échanges avec une tribu voisine. Le massacre des animaux - qui est réalisé avec une violence insoutenable - doit respecter des modalités rituelles sacrificielles pour le rendre acceptable. 
5 La richesse de la documentation ethnographique permet à l'auteur (et au lecteur) de suivre pas à pas les séquences du rituel, dont la plus frappante est celle où l'on expulse les cochons hors de l'espace villageois, en invoquant une sorte de folie "porcine » qui les pousse à s'ensauvager et à se transformer en " ennemis ", car ils sont possédés par les esprits de la forêt. C'est alors que le massacre peut avoir lieu. Cet épisode révèle la construction d'une série de frontières entre l'homme et l'animal, entre les vivants et les morts, entre les humains et les êtres surnaturels, entre les membres de la communauté et les étrangers, entre le chef et ses sujets, entre les hommes et les femmes, entre l'horticulture et l'élevage... En somme, parti d'une réflexion sur la démarcation entre le monde animal et le monde humain, M.M. montre la complexité du processus rituel de construction des limites. La comparaison entre le gâbé Fouyoughé et le kaiko, un rituel pratiqué par une autre société de la Papouasie, permet d'approfondir ces constructions. Mais à la différence du gâbé, le kaiko s'achève par la consommation (interdite chez les Fouyoughé) des cochons domestiques. Des circonstances liées à des contextes historiques peuvent expliquer ces divergences. Le livre s'achève sur une invitation à poursuivre l'analyse de l'élaboration de frontières à partir de rituels modernes qui portent non pas sur une réaffirmation du passé mais sur une attente future.

Il s'agit d'une lecture stimulante qui permet d'apporter un autre éclairage sur les phénomènes religieux et qui invite à poursuivre la comparaison si féconde entreprise par MM. 